\title{
Prevalence and Metabolic Predictors for Early Diagnosed Prediabetes in Women with Previous Gestational Diabetes: Observational Cohort Study
}

\author{
Aleksandra Z. Jotic • Milica M. Stoiljkovic - Tanja J. Milicic • \\ Katarina S. Lalic · Ljiljana Z. Lukic · Marija V. Macesic · \\ Jelena N. Stanarcic Gajovic • Mina M. Milovancevic • Miroslava G. Gojnic Dugalic • \\ Veljko M. Jeremic · Nebojsa M. Lalic
}

Received: July 22, 2021 / Accepted: August 16, 2021 / Published online: August 30, 2021

(C) The Author(s) 2021

\section{ABSTRACT}

Introduction: Women with previous gestational diabetes (pGD) are at higher risk of prediabetes (PD) after delivery. The aim of this study was to determine the prevalence of and predictors for PD among women with pGD.

Methods: The study included 186 women with pGD treated by lifestyle modification. After delivery, the women were divided into group A $(n=80)$ with PD and group B $(n=106)$ with normal glucose tolerance (NGT), defined by the

A. Z. Jotic $(\bowtie) \cdot$ M. M. Stoiljkovic .

T. J. Milicic - K. S. Lalic - L. Z. Lukic ·

M. V. Macesic · J. N. Stanarcic Gajovic .

M. M. Milovancevic · N. M. Lalic

Clinic for Endocrinology, Diabetes and Metabolic Diseases, University Clinical Center of Serbia, Dr. Subotica 13, 11000 Belgrade, Serbia e-mail: aleksandra.z.jotic@gmail.com

A. Z. Jotic - T. J. Milicic - K. S. Lalic .

L. Z. Lukic - M. V. Macesic · J. N. Stanarcic Gajovic ·

M. G. Gojnic Dugalic · N. M. Lalic

Faculty of Medicine, University of Belgrade, Dr.

Subotica 8, 11000 Belgrade, Serbia

M. G. Gojnic Dugalic

Clinic for Gynecology and Obstetrics, University

Clinical Center of Serbia, Visegradska 26,

11000 Belgrade, Serbia

\section{M. Jeremic}

Department for Operations Research and Statistics, Faculty of Organizational Sciences, University of Belgrade, Belgrade, Serbia results of the 2-h oral glucose tolerance test at 4-12 weeks after delivery. We recorded age, body mass index (BMI) at conception and after delivery, fasting glucose (FG), glycated hemoglobin (HbA1c), total cholesterol (TC), triglycerides (Tg), low density lipoprotein cholesterol (LDL-c), high-density lipoprotein cholesterol (HDL-c) and the Tg/HDL-c ratio measured in the third trimester of pregnancy.

Results: Of the 186 women with pGD enrolled in the study, $43 \%$ showed prediabetes at 4 -12 weeks after delivery, with $13.9 \%$ of these women showing impaired FG (IFG), 12.9\% showing impaired glucose tolerance (IGT) and $16.2 \%$ with IFG/IGT. The groups differed in terms of age and BMI at conception and after delivery. In the third trimester of pregnancy, HbA1c was higher in women in group A than in those in group $\mathrm{B}$ (mean \pm standard deviation: $5.6 \pm 0.4$ vs. $5.2 \pm 0.3 \% ; p<0.001$ ), while FG was comparable. Compared to women in group $\mathrm{B}$, women in group A had higher TC $(7.1 \pm 0.8$ vs. $\quad 6.6 \pm 1.0 \mathrm{mmol} / \mathrm{L}), \quad \mathrm{Tg} \quad(2.7 \pm 0.9 \quad$ vs. $2.1 \pm 0.6 \mathrm{mmol} / \mathrm{L})$ and LDL-c $(4.7 \pm 0.8 \mathrm{vs}$. $4.3 \pm 1.0 \mathrm{mmol} / \mathrm{L})($ all $p<0.001)$, lower HDL-c $(1.0 \pm 0.2$ vs. $1.4 \pm 1.0 ; p<0.001)$ and higher median Tg/HDL-c (5.4 [range 4.6-14.3] vs. 4.9 [range 1.1-11.5]; $p<0.001)$. Univariate analysis found an association between prediabetes and age, BMI at conception and after delivery, HbA1c, TC, LDL-c, HDL-c, Tg and Tg/HDL-c ratio. Of these variables, the multivariate analysis showed age (odds ratio [OR] 1.19; 
$p<0.001), \mathrm{HbA1c}(\mathrm{OR} 31.06 ; p<0.001), \mathrm{Tg}$ (OR 4.09; $p<0.001$ ) and LDL-c (OR 2.00; $p=0.005)$ as predictors for prediabetes.

Conclusion: High prevalence of early diagnosed PD in women with pGD was accompanied by advanced age and higher BMI at conception and after delivery. Moreover, age, HbA1c, Tg and LDL-c were predictors for PD.

Keywords: Previous gestational diabetes; Prevalence and metabolic predictors for prediabetes

\section{Key Summary Points}

\section{Why carry out this study?}

Women with previous gestational diabetes (pGD) are at higher risk of prediabetes (PD) after delivery. Despite being the subject of numerous studies, predictors for PD are not clearly defined.

This study was conducted to determine the prevalence of and predictors for PD among women diagnosed with pGD shortly (4-12 weeks) after delivery.

\section{What was learned from the study?}

Our results indicated a high prevalence of PD in women with pGD tested 4-12 weeks after delivery accompanied by advanced maternal age and higher body mass index at conception and after delivery. Advanced age, glycated hemoglobin (HbA1c), triglycerides and low-density lipoprotein cholesterol measured in third trimester were predictors for PD, with $\mathrm{HbA1c}$ as the most prominent predictor.

Predictive accuracy of cutoff value of 5.3\% for HbA1c for detecting PD shortly after delivery among women with pGD showed a rather high sensitivity and specificity.

\section{INTRODUCTION}

Women with previous gestational diabetes (pGD) have a several times higher risk of developing prediabetes and type 2 diabetes (T2D) in future life [1-4]. However, women with pGD seem to be underestimated regarding their future risk of developing prediabetes and T2D. Lifestyle intervention has been shown to have a beneficial effect on the 10-year risk of diabetes in women with a history of pGD and prediabetes [5], which emphasizes the necessity for early screening after delivery.

There is a need for more studies aimed at identifying women with pGD at high risk of prediabetes shortly after delivery who would benefit the most. Earlier studies revealed advanced maternal age, gestational weight gain, fasting plasma glucose (FPG) and glycated hemoglobin (HbA1c) during pregnancy, as well as HbA1c after delivery in being in the shortterm time frame for screening as significant predictors for PB $[1-4,6,7]$. The numerous studies considering $\mathrm{HbA1c}$ and FPG as a predictors of prediabetes after delivery in women with pGD have shown conflicting results [1, 2, 6-8].

Previous studies indicated a specific lipid pattern among women with gestational diabetes (GD) that implies increased levels of triglycerides (Tg), low-density lipoprotein cholesterol (LDL-c) and total cholesterol (TC) and lower levels of high-density lipoprotein cholesterol (HDL-c) most often in late pregnancy $[9,10]$. In contrast, a limited number of investigations hae shown that lipid parameters in early pregnancy might predict GD [11].

In this context, the aim of our study was to evaluate the prevalence of and to identify metabolic predictors for prediabetes within a short time (4-12 weeks) after delivery in women with pGD.

\section{METHODS}

\section{Research Design}

This study included 186 women who were diagnosed as having GD treated only by lifestyle 
modification in pregnancy from 01 January 2017 until 31 December 2019 and followed in the Department for Metabolic Disorders, Intensified Treatment and Cell Therapy in Diabetes, Clinic for Endocrinology, Diabetes and Metabolic Diseases, University Clinical Center of Serbia, Belgrade. All women were followed for glycemic and lipid parameters in pregnancy as well as glucose tolerance $4-12$ weeks after delivery. A 2-h oral glucose tolerance test (OGTT) was conducted 4-12 weeks after delivery, and based on the results the women were placed in one of two groups: group A, comprising those with prediabetes, and group B, comprising those with normal glucose tolerance (NGT), defined according to American Diabetes Association criteria [12]. Prediabetes was defined as impaired fasting glucose (IFG; FPG 5.6-6.9 $\mathrm{mmol} / \mathrm{L}$ ) and/or impaired glucose tolerance (IGT; $7.8-11.0 \mathrm{mmol} / \mathrm{L}$ ) based on the results of a 2-h OGTT after delivery [12]. In all women, we recorded age and body mass index (BMI) before conception (obtained by an interview) and at 4-12 weeks after delivery. We also performed a 2-h, 75-g loaded OGTT at two timepoints: between 24-28 gestational weeks (glucose measured at 0, 60 and $120 \mathrm{~min}$ ) and 4-12 weeks after delivery (glucose measured at 0 and $120 \mathrm{~min}$ ). Data on HbA1c and lipid parameters (TC, LDL-c, HDL-c and $\mathrm{Tg}$ ) were collected in the third trimester of pregnancy in the fasting state.

\section{Inclusion Criteria}

Inclusion criteria were GD according to IADPSG/WHO criteria, where at least one of the glucose values from a 75-g OGTT is equal to or exceeds the following thresholds when treated only by lifestyle modification and singleton pregnancy: FPG, $5.1 \mathrm{mmol} / \mathrm{L}$; 1 -h plasma glucose (PG), $10.0 \mathrm{mmol} / \mathrm{L} ; 2-\mathrm{h} \mathrm{PG}, 8.5 \mathrm{mmol} / \mathrm{L}$ [13].

\section{Exclusion Criteria}

Exclusion criteria were: need for insulin treatment for GD, previous use of metformin, preexisting type 1 diabetes, pre-existing T2D or
T2D diagnosed during 2-h OGTT 4-12 weeks after delivery, pregnancy obtained from assisted reproductive technologies, familial hyperlipoproteinemias (familial hypercholesterolemia and hypertrigliceridemia), overt hyperthyroidism or hypothyroidism, corticosteroid treatment during pregnancy and hepatic disorders.

\section{Measurements}

The relevant parameters were measured as follows:

BMI was calculated according to equation BMI $=$ weight $(\mathrm{kg}) /$ height $\left(\mathrm{m}^{2}\right)$. BMI after delivery was measured before the 2-h OGTT in the fasting state.

HbA1c was measured using a commercial test reagent (SEBIA, Lisses, France).

FPG and PG values during the 2-h OGTT were obtained by the glucose oxidase method using a Beckman Glucose Analyzer (Beckman Instruments Inc., Fullerton, CA, USA).

Serum lipid levels (total and HDL-c and triglycerides) were analyzed enzymatically using a commercial kit (Boehringer Mannheim GmbH Roche Diagnostics, Mannheim, Germany), while LDL-c was calculated by the standard Friedewald formula.

Lipid index (Tg/HDL) was calculated dividing the serum concentration of TG by HDL-c after conversion to $\mathrm{mg} / \mathrm{dL}$ [14].

\section{Ethical Compliance}

This investigation was performed in agreement with both the Declaration of Helsinki of 1964, as revised in 2013, and national guidelines. All participants were informed of the details of the study prior to giving informed consent for participation. All procedures were conducted in accordance with standard clinical settings. The investigation was approved by the Ethics Committee of the Faculty of Medicine, University of Belgrade (1322/VII-19). 


\section{Statistical Analysis}

The descriptive statistics used to characterize the study sample included means, medians and standard deviations (SD) for numerical variables and numbers and percentages for categorical variables. The Kolmogorov-Smirnov and Shapiro-Wilk tests were used to test normal distribution. Associations between categorical data were determined using Pearson's chi-square test. Student's $t$ test or the Mann-Whitney $U$ test were used for numerical data to evaluate the differences between the investigated groups. Univariate and multivariate logistic regression analyses were used to determine factors related to prediabetes. Significant variables from univariate analysis were included in multivariate regressions, with prediabetes as outcome. The results were expressed as relative risk and corresponding 95\% confidence intervals (CI). In all analyses, the level of statistical significance was set at $p \leq 0.05$. The statistical analysis was performed using the SPSS version 21 (2012) software package (SPSS IBM Corp., Armonk, NY, USA).

\section{RESULTS}

The baseline characteristics of the 186 women with pGD are given in Table 1 . Based on the results of the 2-h OGTT performed 4-12 weeks after delivery, $43 \%$ showed prediabetes (group A) and $57 \%$ of women had NGT (group B). Analysis of all women with pGD in the short period after delivery revealed that $13.9 \%$ showed IFG, $12.9 \%$ showed IGT and $16.9 \%$ had IFG/IGT.

There was a significant difference in age (mean \pm SD) at conception between groups A and $\mathrm{B}(36 \pm 5.6$ vs. $34.1 \pm 5.8$ years, respectively; $p=0.023$ ) (Table 1 ). At the same time, compared with women with NGT (group B), those with prediabetes shortly after delivery (group A) had higher BMI before conception $\left(29.2 \pm 6.0\right.$ vs. $\left.27.0 \pm 4.2 \mathrm{~kg} / \mathrm{m}^{2} ; p=0.004\right)$ and after delivery $\left(31.9 \pm 5.0\right.$ vs. $30.1 \pm 3.8 \mathrm{~kg} / \mathrm{m}^{2}$; $p=0.009$ ) (Table 1).

Analysis of the results on glycemic parameters showed that there was no difference in FPG during the third trimester of pregnancy among women with prediabetes $(5.0 \pm 0.5 \mathrm{mmol} / \mathrm{L})$ and those with NGT $(5.0 \pm 0.6 \mathrm{mmol} / \mathrm{L}$; $p=0.825$ ) shortly after delivery (Table 1 ). On the other hand, women with pGD and

Table 1 Baseline characteristics of all women with previous gestational diabetes included in study and according to group based on results of 2 -h oral glucose tolerance test performed at $4-12$ weeks after delivery

\begin{tabular}{llllr}
\hline Baseline characteristics & Total study population $(\boldsymbol{n}=\mathbf{1 8 6})$ & Group A $(\boldsymbol{n}=\mathbf{8 0})$ & Group B $(\boldsymbol{n}=\mathbf{1 0 6})$ & $\boldsymbol{p}$ value \\
\hline Age $($ years $)$ & $34.9 \pm 5.8$ & $36.0 \pm 5.6$ & $34.1 \pm 5.8$ & 0.023 \\
BMI 1 $\left(\mathrm{kg} / \mathrm{m}^{2}\right)$ & $27.9 \pm 5.1$ & $29.2 \pm 6.0$ & $27.0 \pm 4.2$ & 0.004 \\
BMI 2 $\left(\mathrm{kg} / \mathrm{m}^{2}\right)$ & $30.9 \pm 4.4$ & $31.9 \pm 5.0$ & $30.1 \pm 3.8$ & 0.009 \\
HbA1c $(\%)$ & $5.4 \pm 0.4$ & $5.6 \pm 0.4$ & $5.2 \pm 0.3$ & $<0.001$ \\
FPG $(\mathrm{mmol} / \mathrm{L})$ & $5.0 \pm 0.6$ & $5.0 \pm 0.5$ & $5.0 \pm 0.6$ & 0.825 \\
TC $(\mathrm{mmol} / \mathrm{L})$ & $6.8 \pm 0.9$ & $7.1 \pm 0.8$ & $6.6 \pm 1.0$ & $<0.001$ \\
LDL-c $(\mathrm{mmol} / \mathrm{L})$ & $4.5 \pm 0.9$ & $4.7 \pm 0.8$ & $4.3 \pm 1.0$ & $<0.001$ \\
HDL-c $(\mathrm{mmol} / \mathrm{L}$ & $1.2 \pm 0.8$ & $1.0 \pm 0.2$ & $1.4 \pm 1.0$ & $<0.001$ \\
Tg $(\mathrm{mmol} / \mathrm{L})$ & $2.4 \pm 0.8$ & $2.7 \pm 0.9$ & $2.1 \pm 0.6$ & $<0.001$ \\
Tg/HDL-c & $5.1(1.1-14.3)$ & $5.4(4.6-14.3)$ & $4.9(1.1-11.5)$ & $<0.001$ \\
\hline
\end{tabular}

Values in table are presented as the mean \pm standard deviation or as the median with the range in parentheses $B M I 1$ Body mass index at conception, BMI 2 body mass index $4-12$ weeks after delivery 
prediabetes had higher HbA1c in third trimester of pregnancy $(5.6 \pm 0.4 \%)$ compared to women with pGD and NGT shortly after delivery $(5.2 \pm 0.3 \% ; p<0.001)$ (Table 1$)$.

Evaluation of lipid parameters in the third trimester of pregnancy showed that women placed in group A (pGD and prediabetes based on testing shortly after delivery) had higher TC, $\mathrm{Tg}$ and LDL-c than those in group B (NGT shortly after delivery): TC, $7.1 \pm 0.8$ vs. $6.6 \pm 1.0 \mathrm{mmol} / \mathrm{L} ; \mathrm{Tg}, 2.7 \pm 0.9$ vs. $2.1 \pm 0.6$ $\mathrm{mmol} / \mathrm{L} ; \quad$ and $\quad$ LDL-c, $\quad 4.7 \pm 0.8 \mathrm{vs}$. $4.3 \pm 1.0 \mathrm{mmol} / \mathrm{L}$ (all $p<0.001)$ (Table 1$)$. However, HDL-c was lower among women with prediabetes and pGD (group A) than among those with NGT (group B): $1.0 \pm 0.2$ vs. $4 \pm 1.0 \mathrm{mmol} / \mathrm{L} \quad(p<0.001) \quad($ Table 1$) . \quad \mathrm{A}$ between-group difference was also observed for the Tg to HDL-c (Tg/HDL-c) ratio in the third trimester of pregnancy, with higher values observed in group A than in group B (median [range]: 5.4 [4.6-14.3] vs. 4.9 [range 1.1-11.5]; $p<0.001$ ) (Table 1).

Using univariate regression analysis, age and BMI at conception, BMI after delivery, HbA1c, lipid parameters (TC, LDL-c, HDL-c, Tg) and Tg/ HDL-c ratio were found to be significantly associated with prediabetes (Table 2). In the final multivariate regression analysis, four predictors for developing prediabetes shortly (4-12 weeks) after delivery, namely age (odds ratio [OR] $1.19,95 \%$ confidence interval [CI] $1.08-1.32 ; p<0.001$ ), HbA1c (OR 31.06, 95\% CI $7.55-127.8 ; p<0.001$ ), Tg (OR 4.09, 95\% CI $2.01-8.29 ; p<0.001)$ and LDL-c (OR 2.00, 95\% CI 1.23-3.25; $p=0.005)$, remained significant (Table 2).

The predictive accuracy of HbA1c for detecting prediabetes shortly (4-12 weeks) after delivery among women with pGD showed a sensitivity of $77.5 \%(95 \%$ CI $66.8-86.1)$ and specificity of $57.5 \%$ (95\% CI $47.6-67.1)$ for a cut-off of $5.3 \%$ (Area under the curve 0.764, 95\% CI 0.693-0.835; Fig. 1). According to our results, $77.5 \%$ of women with pGD and prediabetes shortly after delivery had HbA1c $\geq 5.3 \%$.

\section{DISCUSSION}

In our observational cohort study, almost half of women with pGD had prediabetes within a short time (4-12 weeks) after delivery. Those identified with prediabetes were older and had higher BMI at conception and after delivery compared to those who were not. Also, we registered higher values of $\mathrm{HbA} 1 \mathrm{c}$ and lipid parameters in the third trimester of pregnancy

Table 2 Logistic regression model with the risk of prediabetes shortly after delivery (4-12 weeks) as dependent variable

\begin{tabular}{|c|c|c|c|c|c|c|c|c|}
\hline \multirow[t]{2}{*}{ Variable } & \multicolumn{4}{|c|}{ Univariate analysis } & \multicolumn{4}{|c|}{ Multivariate analysis } \\
\hline & $\bar{B}$ & SE & OR (95\% CI) & $p$ & $\bar{B}$ & SE & OR $(95 \% \mathrm{CI})$ & $p$ \\
\hline Age & 0.06 & 0.27 & $1.1(1.01-1.12)$ & 0.026 & 0.18 & 0.05 & $1.19(1.08-1.32)$ & $<0.001$ \\
\hline BMI 1 & 0.09 & 0.03 & $1.09(1.03-1.16)$ & 0.005 & & & & \\
\hline BMI 2 & 0.09 & 0.04 & $1.10(1.02-1.18)$ & 0.011 & & & & \\
\hline HbAlc & 2.86 & 0.49 & $17.49(6.66-45.92)$ & $<0.001$ & 3.44 & 0.72 & $31.06(7.55-127.8)$ & $<0.001$ \\
\hline FPG & 0.06 & 0.27 & $1.06(0.63-1.79)$ & 0.824 & & & & \\
\hline $\mathrm{TC}$ & 0.61 & 0.19 & $1.84(1.28-2.66)$ & 0.001 & & & & \\
\hline LDL-c & 0.49 & 0.18 & $1.63(1.14-2.34)$ & 0.008 & 0.69 & 0.25 & $2.00(1.23-3.25)$ & 0.005 \\
\hline HDL-c & -1.46 & 0.48 & $0.23(0.09-0.59)$ & 0.002 & & & & \\
\hline $\mathrm{Tg}$ & 1.08 & 0.26 & $2.94(1.78-4.85)$ & $<0.001$ & 1.41 & 0.36 & $4.09(2.01-8.29)$ & $<0.001$ \\
\hline $\mathrm{Tg} / \mathrm{HDL}$ & 0.27 & 0.07 & $1.39(1.21-1.59)$ & $<0.001$ & & & & \\
\hline
\end{tabular}




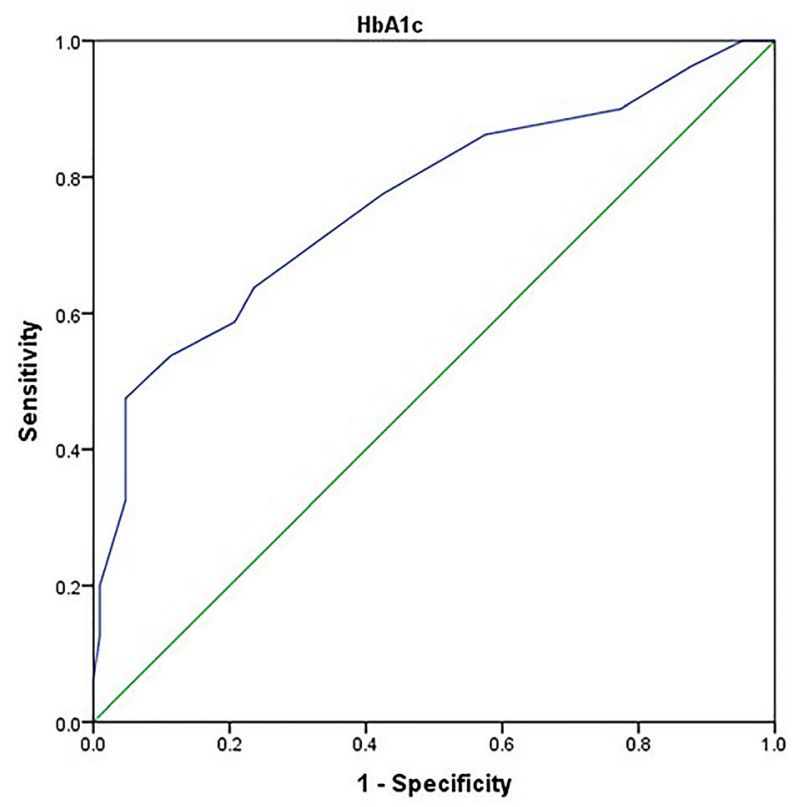

Fig. 1 Predictive accuracy of HbAlc measured in the third trimester of pregnancy for detecting prediabetes shortly (4-12 weeks) after delivery among women with

among women with pGD and prediabetes in the screening time-frame. When we evaluated the risk for prediabetes, age at conception, HbA1c, $\mathrm{Tg}$ and LDL-c were identified as significant predictors.

In clinical settings, post-partum follow-up of pregnant women with pGD is important, as these women have a several-fold increased risk of progression to prediabetes and T2D after delivery $[15,16]$. It is well known that prevalence of prediabetes and T2D among women with pGD varies from 12.7 to $56 \%$ depending on personal characteristics, diagnostic criteria and duration of follow-up [1-4].

Among our study cohort, we found prediabetes within a short period (4-12 weeks) after delivery in $43 \%$ of women with pGD, which is in line with previous results [7]. Interestingly, the incidence rate of early diagnosed diabetes after delivery has been reported to be much higher than the rate of diabetes in long-term follow-up [17]. In one study, despite pGD being known about among some of the pregnant women, only $25 \%$ of those with pGD underwent glucose screening at 4-12 weeks after previous gestational diabetes. Plot shows the ROC curve as well as the respective specificity (57.5\%) and sensitivity (77.5\%) for the HbAlc cut-off level of 5.3\%

delivery [18]. In that context, identifying those women at highest risk of progression from those who remain normoglycemic after GD is of key importance for targeted prevention programs.

Some prior studies in this field have shown that women with pGD who developed prediabetes after delivery were older than those with NGT after delivery [2], which is consistent with our results. In our study, advanced maternal age was associated with a 1.2-fold higher risk of prediabetes, as has previously been found in many different studies [2, 3]. This higher risk may have arisen due to advanced maternal age, suggesting that older pregnant women with pGD should be closely monitored [19].

Previous studies have also pointed out that obesity and gestational weight gain are predictors for prediabetes after delivery among women with pGD $[2,20]$. We also registered higher BMI after delivery, with an average value in the range of obesity in women with pGD and prediabetes within the short period after delivery.

FPG during the third trimester of pregnancy has been evaluated with the aim to diagnose 
glucose intolerance in women with pGD [2]; however, there were conflicting results, with sensitivity rates ranging from 83.0 to $90.0 \%$ [21]. Our results did not show any difference for FPG in the third trimester among women with pGD with respect to glucose tolerance shortly after delivery.

Very few studies have evaluated the clinical usefulness of third-trimester HbA1c levels as a predictor of the development of prediabetes and/or T2D after delivery [2, 22, 23]. In our study, higher HbA1c values were measured in the third trimester in the group of women with pGD who developed prediabetes (group A). Furthermore, multivariate analysis of our data revealed that $\mathrm{HbA1c}$ values in the third trimester were the most prominent predictor for prediabetes after delivery, with women with pGD and higher values of $\mathrm{HbA}_{1} \mathrm{c}$ having a 31-fold increased risk of developing prediabetes in the screening time frame after delivery. The authors of a previous study reported that an HbA1c level $\geq 5.4 \%$, obtained at close to 28 weeks of pregnancy, was associated with a > 5 -fold increased risk of developing diabetes 5 years after pregnancy [8]. When evaluating our data, we defined an optimal cut-off value for the third trimester of pregnancy to be HbA1c < $5.3 \%$, with higher values predicting prediabetes among women with pGD at a sensitivity of $77.5 \%$ and specificity of $57.5 \%$ Although the sensitivity of HbA1c as a screening test to predict prediabetes after delivery in women with pGD appears to be low [8], it may be suitable as a marker that defines high-risk women requiring lifestyle interventions to prevent T2D, starting already in pregnancy and continuing during the woman's lifespan.

The results of the majority of studies conducted to date suggest that women with GD have increased $\mathrm{TC}, \mathrm{Tg}$ and LDL-c levels and lower HDL-c level $[9,10]$. Although it is difficult to ascertain which level of lipid elevation is pathologic, also because there are no defined criteria during pregnancy, we also found higher levels of TC, Tg and LDL-c and lower levels of HDL-c in the group of women with pGD having prediabetes shortly after delivery. From a clinical point of view, hypertriglyceridemia with low HDL-c is a rationale concern as this combination might lead to dysregulation of glucose tolerance [24]. Focusing on higher levels of $\mathrm{Tg}$ as a significant predictor, we showed a fourfold higher risk for prediabetes 4- to 12 -weeks after delivery. This result is in line with a prospective case-control study of women with pGD where the presence of "high-risk" cluster during late pregnancy, defined as a combination of any four cardiometabolic risk factors, including lipids (BMI $>30 \mathrm{~kg} / \mathrm{m}^{2}$, fasting glucose $>5.0 \mathrm{mmol} / \mathrm{L}$, insulin $>7.8 \mathrm{mU} / \mathrm{L}$, $\mathrm{Tg}>2.4 \mathrm{mmol} / \mathrm{L}$, HDL-c $<1.6 \mathrm{mmol} / \mathrm{L}$ or systolic blood pressure $>105 \mathrm{mmHg}$ ) was a better predictor for future T2D development 10 years after delivery than BMI or fasting glucose separately [25].

Some prior studies also found that the $\mathrm{Tg} /$ HDL-c ratio increased significantly throughout the pregnancy among women with GD [10]. In our study, we registered an increased Tg/HDL-c ratio in the third trimester in our group of women with pGD developing prediabetes. However, although univariate analysis showed that the Tg/HDL-c ratio was a significant predictor of $\mathrm{PD}$, this variable was not a significant predictor in the multivariate regression analysis.

In comparison, it is well known that LDL-c is a marker of cardiovascular risk [26], which is substantially higher among women with pGD in the presence of glucose intolerance after delivery [27]. Unexpectedly, we registered higher LDL-c in the third trimester in women with pGD as a significant predictor for the future prediabetes early after delivery (twofold higher risk). However, the results regarding the role of high cholesterol as a risk factor for future glucose intolerance and T2D in women with GD are conflicting. A recent study showed that the lowest TC quartile $(<5.5 \mathrm{mmol} / \mathrm{L})$ at the time of GD diagnosis was associated with an increased risk of abnormal glucose tolerance in women with GD after delivery, compared to lowest risk in the highest TC quartile $(>7.0 \mathrm{mmol} / \mathrm{L})$ group [28]. In contrast, Lappas et al. found significantly higher postnatal TC concentration in women who develop T2D 10 years after GD [29]. In this study, lipidomics analysis of three lipid species, namely, the cholesteryl ester species (ceramide), the alkenylphosphatidylethanolamine species (plasmalogens) 
and the phosphatidylserine species, showed that the levels of these three lipid species were independently and positively associated with the development of T2D in those women with pGD. Ceramide is recognized as a proinflammatory lipid, while plasmalogens are involved in oxidative stress processes [29]. Consequently, LDL enriched in ceramide has been shown to promote inflammation and insulin resistance in skeletal muscle [30] which could partially explain our findings of high LDL-c as a good predictor for the future glucose disturbances in women with pGD. These results reinforce the need for future studies to elucidate the role of TC.

There are two major limitations to our study: (1) its observational study design and (2) a moderate number of included women with pGD related to incidence of GD in our population [31].

\section{CONCLUSIONS}

Our results imply a high prevalence of prediabetes within the short term (4-12 weeks) after delivery among women with pGD, particularly in those of advanced maternal age and with higher BMI at conception and after delivery. Moreover, risk prediction for prediabetes shortly after delivery identified advanced maternal age and available metabolic parameters, emphasizing $\mathrm{HbA} 1 \mathrm{c}$ as the most prominent predictor, followed by $\mathrm{Tg}$ and LDL-c, in the third trimester. Our data should raise awareness of the importance to identify women at the highest risk of prediabetes, starting already in late pregnancy, which would enable the introduction of timely preventive strategies.

\section{ACKNOWLEDGEMENTS}

Funding. The research reported in this article is funded by Project 175097 from the Ministry of Education, Science and Technological Development, Republic of Serbia. No commercial sponsorship was received for this study or publication of this article.
Authorship. All named authors meet the International Committee of Medical Journal Editors (ICMJE) criteria for authorship for this article, take responsibility for the integrity of the work as a whole, and have given their approval for this version to be published.

Author Contributions. Conceptualization: AZ Jotic, MM Stoiljkovic, TJ Milicic, KS Lalic, LZ Lukic, NM Lalić. Methodology: AZ Jotic, MM Stoiljkovic, TJ Milicic, LZ Lukic, MV Macesic, JN Stanarcic Gajovic, MM Milovancevic, MG Gojnic Dugalic, LZ Lukic. Formal analysis and investigation: AZ Jotic, MM Stoiljkovic, TJ Milicic, LZ Lukic, MV Macesic, JN Stanarcic Gajovic, MM Milovancevic, VM Jeremic, NM Lalić. Writing/original draft preparation: AZ Jotic, MM Stoiljkovic, TJ Milicic, KS Lalic, JN Stanarcic Gajovic, NM Lalić. Writing-review and editing: AZ Jotic, MM Stoiljkovic, TJ Milicic, KS Lalic, JN Stanarcic Gajovic, MM Stoiljkovic, NM Lalic. Funding acquisition: NM Lalic. Resources: VM Jeremic, AZ Jotic, TJ Milicic, MM Stoiljkovic, NM Lalic Supervision: AZ Jotic, KS Lalic, NM Lalic.

Disclosures. Aleksandra Z. Jotic is a member of the Diabetes Therapy editorial board. Milica M. Stoiljkovic, Tanja J. Milicic, Katarina S. Lalic, Ljiljana Z. Lukic, Marija V. Macesic, Jelena N. Stanarcic Gajovic, Mina M. Milovancevic, Miroslava G. Gojnic Dugalic, Veljko M. Jeremic and Nebojsa M. Lalic have nothing to disclose.

Compliance with Ethics Guidelines. This investigation was performed in agreement with both the Declaration of Helsinki of 1964, as revised in 2013, and national guidelines. All participants were informed of the details of the study prior to giving informed consent for participation. All procedures were conducted in accordance with standard clinical settings. The investigation was approved by the Ethics Committee of the Faculty of Medicine, University of Belgrade (1322/VII-19).

Data Availability. Access to database is currently strictly regulated by local policy and therefore the data will not be deposited. 
Open Access. This article is licensed under a Creative Commons Attribution-NonCommercial 4.0 International License, which permits any non-commercial use, sharing, adaptation, distribution and reproduction in any medium or format, as long as you give appropriate credit to the original author(s) and the source, provide a link to the Creative Commons licence, and indicate if changes were made. The images or other third party material in this article are included in the article's Creative Commons licence, unless indicated otherwise in a credit line to the material. If material is not included in the article's Creative Commons licence and your intended use is not permitted by statutory regulation or exceeds the permitted use, you will need to obtain permission directly from the copyright holder. To view a copy of this licence, visit http://creativecommons.org/licenses/by$\mathrm{nc} / 4.0 /$.

\section{REFERENCES}

1. Benhalima K, Jegers $\mathrm{K}$, Devlieger $\mathrm{R}$, Verhaeghe J, Mathieu C. Glucose intolerance after a recent history of gestational diabetes based on the $2013 \mathrm{WHO}$ criteria. PLoS ONE. 2016;11:e0157272.

2. Capula C, Chiefari E, Vero A, Foti DP, Brunetti A, Vero R. Prevalence and predictors of postpartum glucose intolerance in Italian women with gestational diabetes mellitus. Diabetes Res Clin Pract. 2014;105:223-30.

3. Shen Y, Wang P, Wang L, et al. Gestational diabetes with diabetes and prediabetes risks: a large observational study. Eur J Endocrinol. 2018;179:51-8.

4. Retnakaran R, Qi Y, Sermer M, Connelly PW, Hanley AJ, Zinman B. Glucose intolerance in pregnancy and future risk of pre-diabetes or diabetes. Diabetes Care. 2008;31:2026-31.

5. Aroda VR, Christophi CA, Edelstein SL, et al. The effect of lifestyle intervention and metformin on preventing or delaying diabetes among women with and without gestational diabetes: the diabetes prevention program outcomes study 10-year follow-up. J Clin Endocrinol Metab. 2015;100: 1646-53.

6. Kojima N, Tanimura K, Deguchi M, et al. Risk factors for postpartum glucose intolerance in women with gestational diabetes mellitus. Gynecol Endocrinol. 2016;32:803-6.

7. Benhalima K, Van Crombrugge P, Moyson C, et al. Prediction of glucose intolerance in early postpartum in women with gestational diabetes mellitus based on the 2013 WHO criteria. J Clin Med. 2019;8:383.

8. Claesson R, Ignell C, Shaat N, Berntorpa K. HbA1c as a predictor of diabetes after gestational diabetes mellitus. Prim Care Diabetes. 2017;11:46-51.

9. Herrera E, Ortega-Senovilla H. Disturbances in lipid metabolism in diabetic pregnancy-are these the cause of the problem? Best Pract Res Clin Endocrinol Metab. 2010;24:515-25.

10. Wang J, Li Z, Lin L. Maternal lipid profiles in women with and without gestational diabetes mellitus. Medicine (Baltimore). 2019;98:e15320.

11. Liu PJ, Liu Y, Ma L, et al. The predictive ability of two triglyceride-associated indices for gestational diabetes mellitus and large for gestational age infant among chinese pregnancies: a preliminary cohort study. Diabetes Metab Syndr Obes. 2020;13: 2025-35.

12. American Diabetes Association. Standards of care. Classification and diagnosis of diabetes: standards of medical care in diabetes. Diabetes Care. 2021;44(Suppl 1):S15-33.

13. Metzger BE, Gabbe SG, Persson B, et al. International association of diabetes and pregnancy study groups recommendations on the diagnosis and classification of hyperglycemia in pregnancy. Diabetes Care. 2010;33:676-82.

14. Masson W, Siniawski D, Lobo M, Molinero G, Huerín M. Association between triglyceride/HDL cholesterol ratio and carotid atherosclerosis in postmenopausal middle-aged women. Endocrinol Nutr. 2016;63:327-32.

15. Vounzoulaki E, Khunti K, Abner SC, Tan BK, Davies MJ, Gillies CL. Progression to type 2 diabetes in women with a known history of gestational diabetes: systematic review and meta-analysis. BMJ. 2020;369:m1361.

16. Bellamy L, Casas JP, Hingorani AD, Williams D. Type 2 diabetes mellitus after gestational diabetes: a systematic review and meta-analysis. Lancet. 2009;373:1773-9.

17. Kim C, Newton KM, Knopp RH. Gestational diabetes and the incidence of type 2 diabetes: a systematic review. Diabetes Care. 2002;25:1862-8. 
18. Nielsen KK, Kapur A, Damm P, de Courten M, Bygbjerg IC. From screening to postpartum followup: the determinants and barriers for gestational diabetes mellitus (GDM) services, a systematic review. BMC Pregnancy Childbirth. 2014;14:41.

19. Jolly M, Sebire N, Harris J, et al. The risks associated with pregnancy in women aged 35 years or older. Hum Reprod. 2000;15:2433-7.

20. Wahabi H, Fayed A, Tunkar SMS, et al. Incidence and contributing factors of glucose intolerance in Saudi postpartum women: Sub-group analysis from RAHMA study. PLoS ONE. 2019;14:e0210024.

21. Noctor E, Crowe C, Avalos G, Kirwan B, O'Shea PM, Dunne FP, for the ATLANTIC DIP investigators. ATLANTIC DIP-Simplifying the follow-up screening for women with previous gestational diabetes (GDM): a population-based study. Eur J Endocrinol. 2013;169:681-7.

22. Liu H, Zhang S, Wang L, et al. Fasting and 2-hour plasma glucose, and HbA1c in pregnancy and the postpartum risk of diabetes among Chinese women with gestational diabetes. Diabetes Res Clin Pract. 2016;112:30-6.

23. Kwon SS, Kwon JY, Park YW, Kim YH, Lim JB. $\mathrm{HbA1c}$ for diagnosis and prognosis of gestational diabetes mellitus. Diabetes Res Clin Pract. 2015;110:38-43.

24. Parhofer K. Interaction between glucose and lipid metabolism: more than diabetic dyslipidemia. Diabetes Metab J. 2015;39:353-62.
25. Barden A, Singh R, Walters B, Phillips M, Beilin LJ. A simple scoring method using cardiometabolic risk measurements in pregnancy to determine 10-year risk of type 2 diabetes in women with gestational diabetes. Nutr Diabetes. 2013;3:e72.

26. Bertoluci MC, Rocha VZ. Cardiovascular risk assessment in patients with diabetes. Diabetol Metab Syndr. 2017;20(9):25.

27. Burlina S, Dalfrà MG, Lapolla A. Gestational diabetes mellitus and future cardiovascular risk: an update. Int J Endocrinol. 2016;2016:2070926.

28. Wang D, Ding W, Xu S, Chen H, Liu B, Wang Z. The relationship between total cholesterol and postpartum impaired glucose tolerance in women with gestational diabetes mellitus. Lipids Health Dis. 2020;19:142.

29. Lappas M, Mundra PA, Wong G, et al. The prediction of type 2 diabetes in women with previous gestational diabetes mellitus using lipidomics. Diabetologia. 2015;58:1436-42.

30. Boon J, Hoy AJ, Stark R, et al. Ceramides contained in LDL are elevated in type 2 diabetes and promote inflammation and skeletal muscle insulin resistance. Diabetes. 2013;62:401-10.

31. Savona-Ventura C, Vassallo J, et al. A composite risk assessment model to screen for gestational diabetes mellitus among Mediterranean women. Int J Gynaecol Obstet. 2013;120:240-4. 